Research Article

\title{
Neuroprotective Effect of Monosialotetrahexosylganglioside (GM1) on Patients with Parkinson's Disease Anesthetized by Ketamine under Denoising Algorithm-Based Ultrasound Image Diagnosis
}

\author{
Weili Wang $\mathbb{D}$, ${ }^{1}$ Mingwei Huang $\mathbb{D},{ }^{1}$ Tingting Lin $\mathbb{D},{ }^{2}$ Chengzhi Lu $\mathbb{D}$, ${ }^{1}$ and Jiandong Liu $\mathbb{D}^{1}$ \\ ${ }^{1}$ Department of Anesthesiology, 909th Hospital of PLA (Affiliated Dongnan Hospital of Xiamen University), Zhangzhou 363600, \\ Fujian, China \\ ${ }^{2}$ Department of Outpatient, 909th Hospital of PLA (Affiliated Dongnan Hospital of Xiamen University), Zhangzhou 363600, \\ Fujian, China
}

Correspondence should be addressed to Jiandong Liu; iabp@mail.sdufe.edu.cn

Received 18 September 2021; Revised 1 December 2021; Accepted 3 December 2021; Published 23 December 2021

Academic Editor: M Pallikonda Rajasekaran

Copyright (c) 2021 Weili Wang et al. This is an open access article distributed under the Creative Commons Attribution License, which permits unrestricted use, distribution, and reproduction in any medium, provided the original work is properly cited.

This study was to investigate the value of ultrasound technology based on the bilateral filtering noise elimination algorithm in evaluating the neuroprotective effect of monosialoganglioside in ketamine-anesthetized Parkinson's disease patients. The research subjects were 75 patients with Parkinson's disease admitted to the hospital. The patients were randomly divided into three groups according to different treatment methods: A (GM1 + ketamine anesthesia group), B (conventional treatment + ketamine anesthesia group), and C (GM1 + nonketamine anesthesia group), with 25 patients in each group. Twenty-five healthy people with similar general data in the three groups (groups A, B, and C) were also selected as the control group (group D). All patients underwent ultrasonography, and ultrasound images were processed using the bilateral filter noise elimination. Structural similarity (SSIM), mean absolute error (MAE), and peak signal to noise ratio (PSNR) were used to evaluate the treatment effect. Plasma phospholipids, the third part of the PD unified score scale, Montreal cognitive assessment scale, and other indicators were analyzed and compared among the four groups. The bilateral filtering image noise was effectively suppressed, and the edge details were kept well. Some of the weak edges and texture information in the image were eliminated, the visual effect was ideal, and the accuracy of the edges of the picture remained good. The serotonin lipid level in group A was greatly lower than the serum phospholipid level in group B after GM1 treatment (6.55 VS 7.84, $P<0.05)$. Compared with that before treatment, the serotonin lipid level of group A patients decreased after the treatment, and the difference was considerable $(7.46 \mathrm{VS} 6.55, P<0.05)$. In short, GM1 had a protective effect on the nerves of patients with Parkinson's disease anesthetized by ketamine.

\section{Introduction}

Parkinson's disease, also known as paralysis tremors, is another common neurodegenerative disease in addition to Alzheimer's disease and is a chronic neurodegenerative disease [1]. According to the world survey of neurological diseases, people about $0.3 \%$ of developed countries have Parkinson's disease [2]. Parkinson's disease affects about one percent of older people as they get older and about four percent of older people between ages 60 and 80 . Age is a major risk factor for Parkinson's disease. The specific onset of Parkinson's disease has its own characteristics. In addition, gender is a factor for Parkinson's disease, which may be related to female hormones $[3,4]$.

Ketamine, whose chemical name is chlorobenzenemethyl amino cyclohexane, is a derivative of the phenyl epoxy group, which is a kind of phencyclidine derivatives. Originally synthesized to replace phencyclidine (PCP), it is an effective n-methyl-d-aspartic acid (NMDA) receptor blocker. In the 1970s, it was used clinically and widely in 
various operations requiring anesthesia [5]. Ketamine is not only used in animals but also in the treatment of humans during and after surgery. Ketamine is not only used in anesthesia for Parkinson's disease but also plays an important role in other neurological diseases [6].

Gangliosides (GLS) are a type of sialic acid glycosphingolipids. It is found in all kinds of tissues in mammals, mainly in the central nervous system, and it is high in the brain. Astrocytes and oligodendrocytes also contain gangliosides [7-9]. Ganglioside has four categories, namely, single sialic acid ganglioside monosialotetrahex osylganglioside (GM1), GD1a, GD1b, and GT1b. GM1 accounts for the largest proportion and has been studied the most [10]. GM1 consists of a hydrophobic ceramide and a hydrophilic sugar chain and contains a molecule of sialic acid [11]. Studies revealed that GM1 can play a neuroprotective role in a variety of conditions, including the survival of nerve cells, the promotion of synaptic growth of nerve cells, the promotion of neuronal differentiation, and the protection of various types of nerve cells [12].

There are many forms of diagnosis commonly used in medical diagnosis, and $\mathrm{B}$ ultrasound is a very common method. Ultrasonic imaging technology, X-ray diagnostic technology, and isotope technology have been identified as the three latest medical imaging technologies [13]. It is widely used in the pathological diagnosis of cardiovascular disease, the liver, spleen, gallbladder, pleura, and the other parts of the human body and also plays an important role in the diagnosis of fetal morphology in obstetrics department. It has the advantages of radiation damage, economy, clear imaging, and painless diagnosis. As an important optimization method for the B-ultrasonic imaging system, image processing plays an important role in the image quality and is directly related to the performance of the entire equipment [14]. In the ultrasonic diagnostic device, there is the starting receiving part of the front-end system, A/D conversion module, and beam synthesis technology, which are accompanied by the image transmission module and operation. Therefore, when the outcome is usually affected by external noise, the generated images also affect doctors' diagnosis [15]. The resolution of the image is reduced, the image edge recognition is not clear, and the image quality is affected, which will cause a great interference to the disease diagnosis. Linear region filtering performs a weighted average calculation of pixels based on the gray value or position relationship of block pixels. Spatial filtering has a communication port, which also determines the filtering effect. The size of the communication port determines the quality of the nearby relevant pictures. The finer the pixel, the better the quality of the picture. At present, the most commonly used is wavelet threshold noise elimination [16]. To optimize the noise cancellation, the selection of wavelet threshold noise cancellation is optimized, and the adaptive threshold wavelet noise cancellation is proposed. Wavelet correlation noise elimination correlates the wavelet coefficients of each layer with the wavelet coefficients of the upper and lower layers. However, the noise in the image does not have such a relationship, and noise removal is performed through the wavelet property of adjacent scales [17].
In this study, Parkinson's patients were studied, and ultrasound techniques based on bilateral filtered noise elimination algorithms were used to diagnose patients. On this basis, the neuroprotective effect of GM1 on ketamineanesthetized Parkinson's disease patients was explored from the plasma phospholipid level, the third part of the PD unified scoring scale, Montreal cognitive assessment scale, and other indicators, in order to provide reference and basis for the treatment of related diseases in clinical practice.

\section{Methods}

2.1. Research Objects. The research subjects were based on 75 patients with Parkinson's disease admitted to the hospital from May 2019 to May 2021. Inclusion criteria: (i) patients with Parkinson's disease, (ii) brain imaging was performed using ultrasound, (iii) patients with Parkinson's disease anesthetized by ketamine, (iv) patients had no other brain disease, (v) patients had no other neurological disease. Exclusion criteria: (i) nonparkinson's disease; (ii) patients under 25 years of age; (iii) patients with poor compliance; (iv) patients with other brain diseases or complications other than hydrocephalus (such as cerebral hemorrhage and encephalitis). The patients were randomly divided into three groups according to different treatment methods: A (GM1 + ketamine anesthesia group), B (conventional treatment + ketamine anesthesia group), and C (GM1 + nonketamine anesthesia group), with 25 patients in each group. Twenty-five healthy people with similar general data in the three groups (groups A, B, and C) were also selected as the control group (group D). In this study, a total of 100 Parkinson's patients who met the above inclusion criteria and exclusion criteria were included. This study had been approved by the ethics committee of the hospital, and all the families of the patients included in the study had signed the informed consent.

2.2. Denoising Algorithm. The main idea of the bilateral filtering noise elimination is using a weighted average of nearby pixels very similar to Gaussian convolution. The advantage of this algorithm is that it can smoothen and maintain the flatness of the image edge, considering the difference in the gray value of the bilateral filter at the surrounding position points. To summarize the mechanism of bilateral filtering, the gray value of a pixel depends on the surrounding pixels. It is necessary that two pixels are not only adjacent in the spatial position but also have similar gray values. The bilateral filtering equation is as follows:

$$
\mathrm{BF}[I]_{P}=\frac{1}{W_{P}} \sum_{q \in S} G_{\sigma_{d}}(\|p-q\|) G_{\sigma},\left(I_{p}-I_{q}\right) \cdot I_{q} .
$$

$W_{P}$ is the normalization factor, and the equation is as follows:

$$
W_{p}=\sum G_{\sigma_{d}}(\|p-q\|) G_{\sigma r}\left(I_{p}-I_{q}\right) .
$$

$\sigma_{d}$ and $\sigma_{r}$ are related to the image filter value. $G_{\sigma_{d}}$ acts on the farther pixels and can reduce the influence of noise. $G_{\sigma r}$ 
affects adjacent pixels, which can also reduce the influence of noise. The Gaussian function is used to define $G_{\sigma_{d}}$ and $G_{\sigma r}$. They are both spatial functions and functions to measure pixels. The equations are expressed as follows:

$$
\begin{aligned}
& G_{\sigma_{d}}=e^{-(1 / 2)\left(d(p, q) / \sigma_{d}\right)^{2}}, \\
& G_{\sigma r}=e^{-(1 / 2)(\delta(I(p), I(q)) / \sigma)^{2}} .
\end{aligned}
$$

$d(p, q)$ is the straight-line distance between two points on the picture. $\delta(I(p), I(q))$ is the pixel difference between two points $p$ and $q$; this difference affects the denoising ability of the bilateral filter. The larger the $\sigma_{d}$, the more noise in the image and the more blurred the image. $G_{\sigma r}$ is also calculated in the same way, so the more noise, the more blurred the image.

2.3. Ultrasound Image Noise Reduction Effect. The image noise reduction effect is assessed by subjective evaluation and objective evaluation. Subjective evaluation refers to observing the noise reduction effect with the naked eye, while the objective evaluation is indicated by data. SSIM is the ratio of the same characteristics of the picture's structure, which shows the contrast of the quality and fineness of the picture before and after compression. To calculate SSIM, it needs to compare two pictures at the same time and calculate the identity and difference between the two pictures. The picture size is set to $x$ and $y$, and the equation is as follows:

$$
\operatorname{SSIM}(x, y)=\frac{\left(2 \alpha_{x} \alpha_{y}+c_{1}\right)\left(2 \sigma_{x y}+c_{2}\right)}{\left(\alpha_{x} \alpha_{y}+c_{1}\right)\left(\sigma_{x}^{2}+\sigma_{y}^{2}+c_{2}\right)}
$$

In the equation, the variance of the pixels in the window is $\sigma^{2}$, and $C_{1}$ and $C_{2}$ in the picture represent the average value of the gray values of the pixels, that is, the dispersion of the gray values of the pixels of the image. The larger the SSIM value, which is the covariance of image blocks $x$ and $y$, the more similar the structural information between the noiseremoved image and the original image.

The mean absolute error (MAE) and the peak signal to noise ratio (PSNR) are calculated as follows:

$$
\begin{aligned}
\text { PSNR } & =10 \log _{10}\left(\frac{255^{2} \times M \times N}{\sum_{w=1}^{M} \sum_{e=1}^{N} \mid u(w, e)-f(w, e)^{2}}\right), \\
\text { MAE } & =\frac{\sum_{w=1}^{M} \sum_{e=1}^{N} \mid u(w, e)-f(w, e)}{M \times N} .
\end{aligned}
$$

$M \times N$ represents the size of the image. $u$ and $f$ are the original image and the noise-removed image, respectively. The higher the PSNR value, the smaller the MAE, and the better the image noise reduction effect.

\subsection{Clinical Observation Index Plasma Phospholipid Level.} The test subjects were selected to detect the content of blood phospholipids, and the results included the identification of the quality of blood phospholipids. All subjects avoided abnormal lifestyles the day before plasma phospholipids were tested. Patients with menstrual period and fever needed to delay detection until the recovery day. $5 \mathrm{ml}$ venous blood was collected on fasting. After the blood was collected, the blood was put into an anticoagulation test tube and centrifugated for 20 minutes $(800 \mathrm{r} / \mathrm{min})$. After centrifugation, the phospholipids were quantified with $1 \mathrm{ml}$ of topmost plasma. Blood phospholipids were determined by colorimetry combined with a quantitative test of modified inorganic phosphorus, and the test results were expressed in unit $\mathrm{u}$. The main steps were extracting the phospholipid, detecting the concentration of the sample, separating, staining, taking a water bath at $90^{\circ} \mathrm{C}$ for $5 \sim 10$ minutes, cooling it to room temperature, and determining the quantity after 30 minutes.

2.5. UPDRS-III Scoring. Patients in groups A and B received the unified Parkinson's disease rating scale part III (UPDRSIII) before and after drug intervention, which includes the following 14 aspects of language (score 0-4), facial expression, static signs, handshaking or postural signs, muscular tension, symptoms of fingers and knees, hand movements, rapid alternating hand movements, knee bending, range of motion of lower limbs, lifting ability of arms, stability of gait, slow bending position, and scores on each side (score 0-4). The higher the score, the more developed the patient's motor nerve.

2.6. MOCA Scale Examination. Patients in groups A and B received the Montreal cognitive assessment scale (MOCA) test before and after the drug intervention. MOCA consists of the following components of visuospatial and executive action (one to five), language function (a 6-point scale from one to six that evaluates the patient's language ability), directions (on a scale of one to six), memory (on a scale of one to six), sensitivity to numbers (on a scale of one to four), and abstraction of graphics (on a scale of one to two). To assess cognitive function and the degree of cognitive dysfunction, the evaluation process was performed by two physicians selected from the medical school.

2.7. Statistical Analysis. SPSS 20.0 was used for statistical analysis. Normally distributed measurement data were expressed as mean plus or minus standard deviation. Oneway analysis of variance was used for intergroup comparison. An independent sample $t$-test was used for general data. Paired sample $t$-test was used to compare lung LUS scores of healthy volunteers at different time points. $P<0.05$ meant that the difference was statistically considerable.

\section{Results}

3.1. Visual Comparison of Efficiency of Natural Noise Reduction and BF Image Noise Reduction. Different ultrasound images were denoised in the natural image simulation experiment. To obtain the noise image, the average value 0 and Gaussian noise 0.002 were added to the test image, respectively, and the noise reduction experiment was carried out on the noise image. For the noise removal ability of BF, 
the subjective visual evaluation and objective evaluation indicators MAE, PSNR, and MSSIM were used to evaluate and analyze the image quality with reduced noise. The parameters of each comparison model in the experiment were set according to the best experimental results. The subjective visual effects are shown in Figure 1, and the comparison of objective evaluation performance indicators is shown in Figure 2. Analysis of Figure 2 showed that the values of MSSIM, PSNR/dB, and MAE of ultrasound images processed by the BF noise reduction algorithm were 0.915 , 32.7, and 4.2, respectively; the values of MSSIM, PSNR/dB, and MAE of ultrasound images processed by the conventional noise reduction algorithm were $0.86,32$, and 4.43 , respectively; and the values of MSSIM, PSNR/dB, and MAE of original ultrasound images were $0.84,31.75$, and 4.66, respectively. The performance of the $\mathrm{BF}$ noise reduction algorithm in ultrasonic image processing was better than that of the conventional noise reduction algorithm.

In the normal noise reduction process shown in Figure 1, the edge information of the image was well preserved, but the resulting noise had a poor visual effect. The BF processing result had a good visual effect, the image noise was effectively suppressed, and the edge details were maintained well. Some weak edges and texture information in the image were eliminated, the visual effect became better, and the accuracy of the edges of the picture remained good.

3.2. Comparison of Plasma Phospholipid Levels in the Four Groups of Subjects before Intervention. The blood phospholipid levels of the four groups before the drug intervention were measured and compared. The blood phospholipid levels of the four groups before intervention were $7.46,7.88,5.38$, and 4.72 , respectively (Figure 3 ). There was no considerable difference in blood phospholipid levels between the two groups $(P>0.05)$. The plasma phospholipid levels of group A and group B were greatly higher than group C. The level of blood phospholipids in group D was greatly lower than in the first three groups. The results showed that Parkinson's patients had cell membrane damage, and their blood phospholipid levels were higher than normal. The increase in blood levels of small phospholipids in Parkinson's patients was associated with the use of ketamine anesthesia. Plasma phospholipid concentrations were high in ketamine-anesthetized Parkinson's patients. Therefore, the plasma phospholipid level can be used as a potential biochemical marker to study the early diagnosis of GM1 in patients with Parkinson's anesthesia with ketamine.

3.3. UPDRS-III and MOCA Scores of Patients in Groups A and $B$ before Intervention. Before treatment with GM1, AB group patients were scored on the UPDRS-III and MOCA scales. UPDRS-III and MOCA scores were 21.74 and 22.17 in group A and 20.65 and 25.66 in group B, respectively. From the comparison of the scores of group A and group B, there was no considerable difference in UPDRS-III, and there was a considerable difference in the interference of MOCA by drugs $(P>0.05)$. It showed that the severity of motor symptoms and cognitive dysfunction of the two groups of patients before receiving the intervention was similar and comparable (Figure 4).

3.4. UPDRS-III and MOCA Scores after GM1 Treatment of Ketamine Anesthesia in Parkinson's Patients. After GM1 was adopted to treat ketamine-anesthetized Parkinson's patients, the UPDRS-III and MOCA scores were recorded again and compared with the previously measured ones (Figures 5 and 6). After treatment, the MOCA and UPDRS-III scores in group A were 23.88 and 20.93, respectively, and the MOCA and UPDRS-III scores in group B were 22.13 and 20.27, respectively. In addition, the MOCA score was greatly different between the two groups before and after GM1 treatment. Compared with that before treatment, the MOCA score of group A was not greatly different from the previous record $(P>0.05)$. Compared with that before GM1 treatment, the MOCA score of group B decreased after treatment, with a considerable difference $(P<0.05)$. At the end of treatment, the MOCA score of group A increased greatly $(P<0.05)$, and there was no considerable difference in UPDRS-III scores. The results showed that GM1 can slow down the speed of nerve paralysis in Parkinson's patients anesthetized by ketamine and have a protective effect on the brain nerves of Parkinson's patients. The specific results are shown in Figure 7.

3.5. Plasma Phospholipid Levels of Two Groups of Patients after GM1 Treatment of Ketamine Anesthesia for Parkinson's Patients. After six weeks of treatment, the blood phospholipid levels of the AB groups were measured again. At the end of treatment, the blood phospholipids of group A and group B were 6.55 and 7.84, respectively (Figure 7). Compared with the blood phospholipid level before GM1 treatment, the serotonin lipid level in group A was greatly lower than the serum phospholipid level in group B after GM1 treatment $(P<0.05)$. Compared with that before treatment, the serotonin lipid level of patients in group A decreased after treatment, and the difference was considerable $(P<0.05)$. It showed that GM1 treatment can reduce the serotonin lipid level and protect the cell membrane of nerve cells.

\section{Discussion}

According to the World Survey of Neurological Diseases, about $0.3 \%$ of developed countries have Parkinson's disease. With the increase of age, one percent of the elder people have Parkinson's disease, and four percent of the elder people aged 60-80 have Parkinson's disease. Age is a major risk factor for Parkinson's disease [18]. Anesthesia plays an important role in the diagnosis and treatment of Parkinson's disease, but improper anesthesia can cause serious consequences, and the whole body may have fatal reactions after general anesthesia. The improper use of anesthesia also causes irreversible damage to the central spirit of the brain. During the rapid development of the brain, the number of synapses is developing and maturing [19]. Finding a 


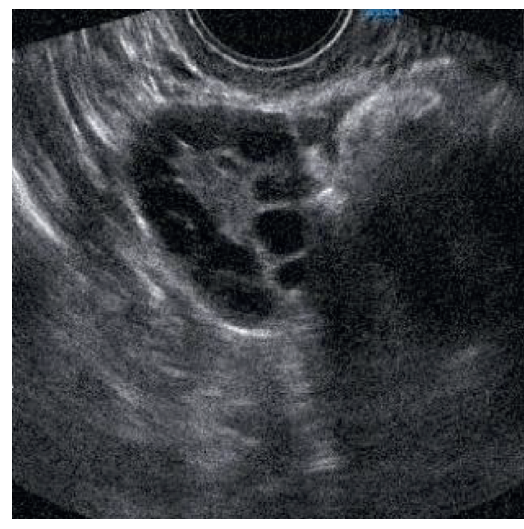

(a)

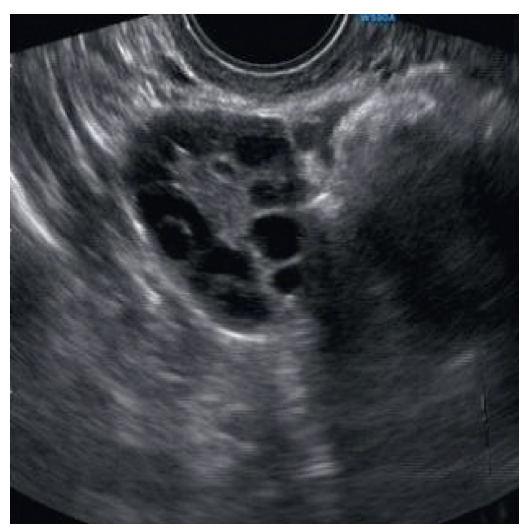

(b)

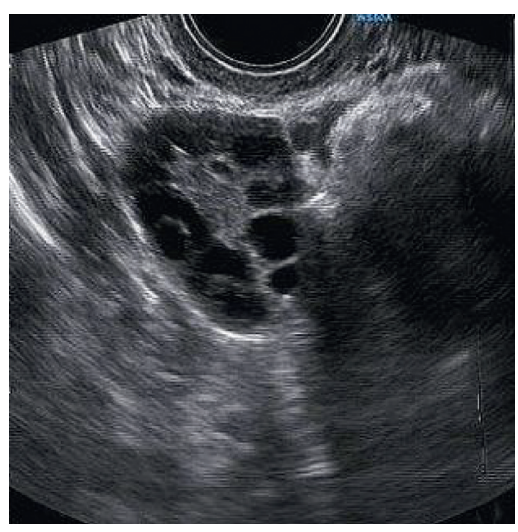

(c)

FIGURE 1: Visual comparison diagram after different methods of noise reduction processing. (a) original ultrasonic image; (b) conventional noise reduction ultrasonic image; (c) BF noise reduction ultrasonic image.

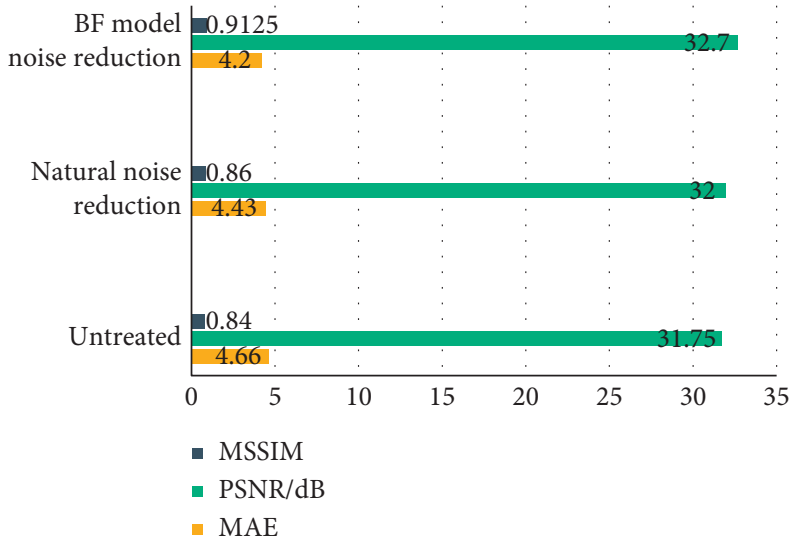

FIGURE 2: Comparison of objective performance evaluation indicators corresponding to unprocessed, normal noise reduction, and BF noise reduction.

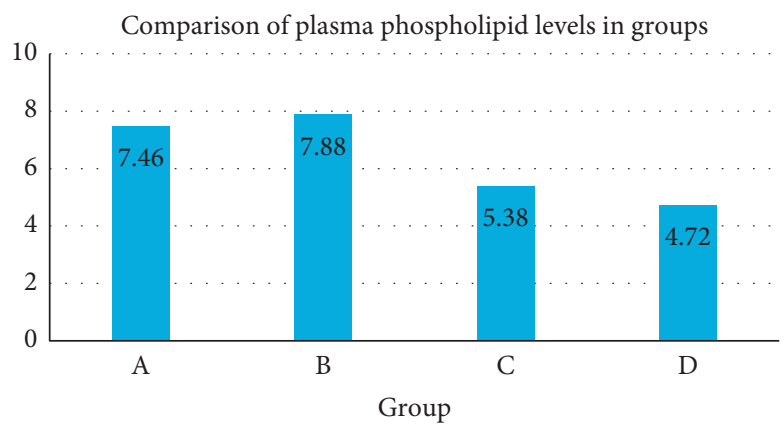

Figure 3: Comparison of plasma phospholipid levels of subjects in groups A, B, C, and D before treatment.

substance that protects nerves damaged by anesthesia in the brain is crucial.

At present, the most common anesthetic drug in clinical practice is ketamine. However, a large number of clinical studies showed that ketamine anesthesia can induce neuronal apoptosis in the developing brain, causing damage to the patient's brain and not conducive to the patient's recovery. Therefore, in recent years, scientists have been working on excavating drugs that can reduce ketamine toxicity. At present, some progress has been made in related research. GM1 is one of the important substances that has attracted much attention. GM1 consists of a hydrophobic ceramide and a hydrophilic sugar chain, which contains a molecule of sialic acid. Its hydrophilic groups are outside the cell membrane, and the hydrophobic group GM1 plays a variety of roles in the physiology and pathology of nerve 


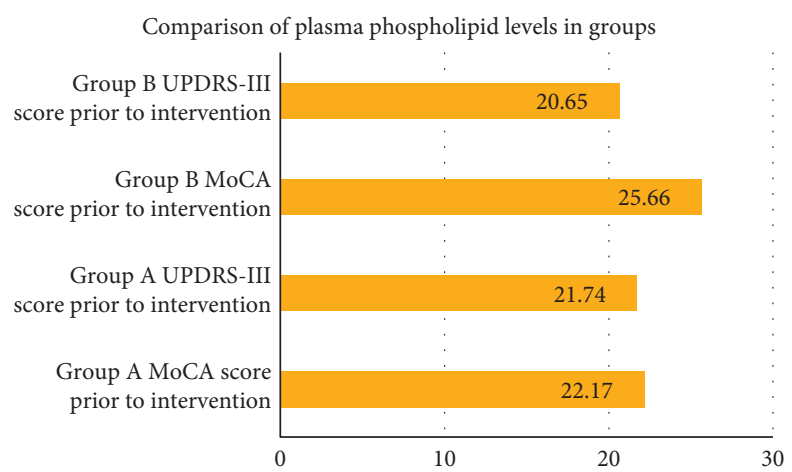

FIGURE 4: UPDRS-III and MOCA scores of patients in groups A and B before using GM1.

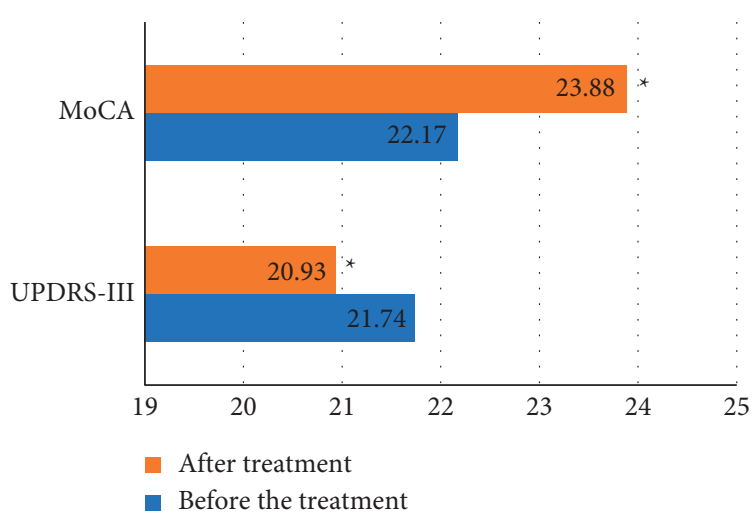

FIgURE 5: Comparison of UPDRS-III and MOCA scores before and after treatment in group A. ${ }^{*} P<0.05$ compared with that before treatment.

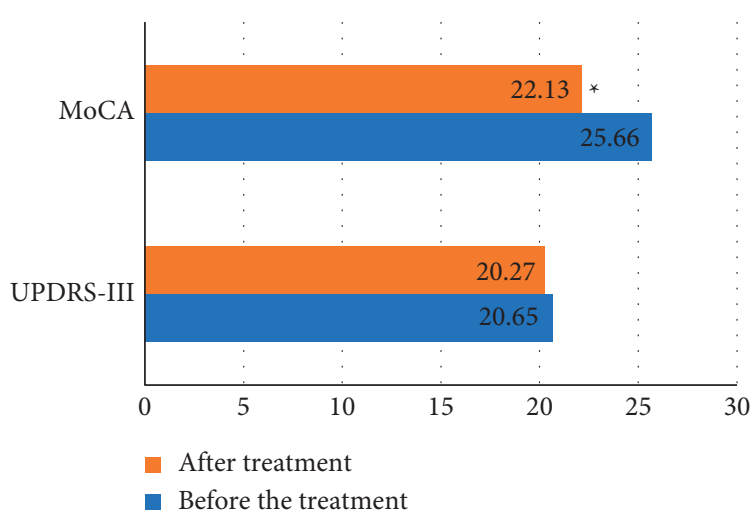

Figure 6: Comparison of UPDRS-III and MOCA scores before and after treatment in group B. ${ }^{*} P<0.05$ compared with that before treatment.

cells. A large number of biological and clinical studies indicated that GM1 can not only effectively reduce the toxicity of ketamine to the brain but also relieve parkinsonian symptoms [20]. Although some progress has been made in the current research on GM1 to reduce ketamine toxicity and relieve parkinsonian symptoms, it is not comprehensive and in-depth enough.

Many clinical studies suggested that Parkinson's patients mostly have metabolic disorders in the nervous system, with abnormal deposition of iron, most significantly in the substantia nigra. Transcranial ultrasound can detect hyperechoic signals in the substantia nigra. Based on the above characteristics, cranial ultrasound was widely used in the diagnosis of Parkinson's disease and the evaluation of efficacy. With the more and more widespread use of ultrasound technology and the more and more profound understanding of diseases [21], the quality requirements for ultrasound images have been further improved. Traditional 


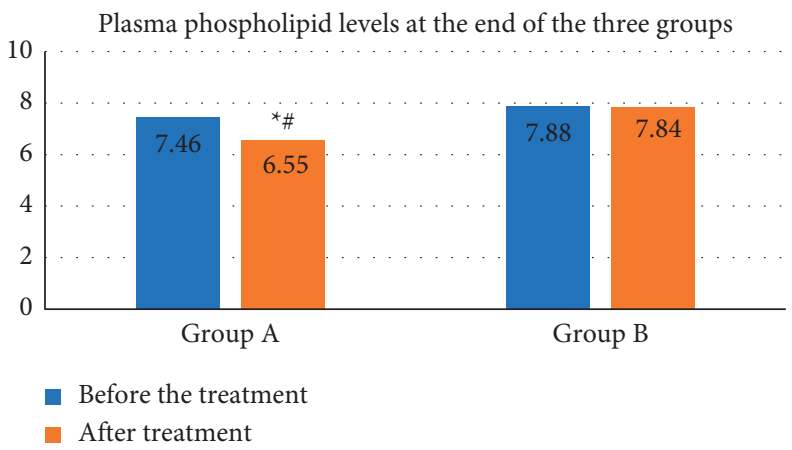

Figure 7: The blood phospholipid levels of patients in the groups A and B after GM1 treatment with ketamine anesthesia. ${ }^{*} P<0.05$, compared with that before treatment. ${ }^{\#} P<0.05$, compared with group B.

image processing technology was unable to meet people's needs. In recent years, computer and Internet technology has been continuously advanced and developed. Medical image processing technology has also been greatly developed. Many image processing algorithms have emerged. According to the characteristics of more noise in ultrasonic images, the bilateral filtered noise elimination algorithm is most widely used in the processing of ultrasonic images, for example, the reconstruction of three-dimensional ultrasound images using bilateral filtering noise elimination algorithms. However, there is little application of bilateral filtered noise elimination algorithms in ultrasound image processing in Parkinson's patients [22].

In this study, Parkinson's patients were selected as the study subjects, and ultrasound technology based on the bilateral filtered noise elimination algorithm was used to diagnose the patients and explore the neuroprotective effect of GM1 in ketamine-anesthetized Parkinson's disease patients on this basis. It was found that the processing results of BF have a good visual effect, the image noise is effectively suppressed, the details of the edge are well maintained, some weak edges and texture information in the image are eliminated, the visual effect becomes better, and the accuracy of the edge of the picture is well maintained. The blood phospholipids of groups A and B were $6.55,7.84$, and 5.39 , respectively. Blood phospholipid levels were compared with those before GM1 treatment, and serotonin levels in group A were significantly lower than serum phospholipid levels in group B after GM1 treatment $(P<0.05)$. Serotonin levels decreased after treatment in group A patients compared to before treatment, and the difference was statistically significant $(P<0.05)$.

\section{Conclusion}

In this study, Parkinson's patients were diagnosed by the ultrasound technique based on the bilateral filtered noise elimination algorithm, and the neuroprotective effect of GM1 in ketamine-anesthetized Parkinson's disease patients was explored on this basis. The results of the study showed that GM1 relieved parkinsonian symptoms and ketamine neurotoxicity. The bilateral filtered noise elimination algorithm has a better performance in ultrasound image processing in Parkinson's patients. Therefore, the bilateral filtered noise elimination algorithm and GM1 have high application value in neuroprotection of ketamine-anesthetized Parkinson's disease patients. Due to the limited sample and length, this study is not comprehensive and has no in-depth research. In the future study and work, it will expand the sample to study this problem further comprehensively and deeply.

\section{Data Availability}

The data used to support the findings of this study are available from the corresponding author upon request.

\section{Conflicts of Interest}

The authors declare no conflicts of interest.

\section{References}

[1] E. R. Dorsey, T. Sherer, M. S. Okun, and B. R. Bloem, "The emerging evidence of the Parkinson pandemic," Journal of Parkinson's Disease, vol. 8, no. s1, pp. S3-S8, 2018.

[2] R. B. Schneider, J. Iourinets, and I. H. Richard, "Parkinson's disease psychosis: presentation, diagnosis and management," Neurodegenerative Disease Management, vol. 7, no. 6, pp. 365-376, 2017.

[3] N. Titova, C. Padmakumar, S. J. G. Lewis, and K. R. Chaudhuri, "Parkinson's: a syndrome rather than a disease?" Journal of Neural Transmission, vol. 124, no. 8, pp. 907-914, 2017.

[4] S. Lotankar, K. S. Prabhavalkar, and L. K. Bhatt, "Biomarkers for Parkinson's disease: recent advancement," Neuroscience Bulletin, vol. 33, no. 5, pp. 585-597, 2017.

[5] P. Zanos and T. D. Gould, "Mechanisms of ketamine action as an antidepressant," Molecular Psychiatry, vol. 23, no. 4, pp. 801-811, 2018.

[6] P. Zanos, R. Moaddel, P. J. Morris et al., "Ketamine and ketamine metabolite pharmacology: insights into therapeutic mechanisms," Pharmacological Reviews, vol. 70, no. 3, pp. 621-660, 2018.

[7] R. Ledeen and G. Wu, "Gangliosides of the nervous system," Gangliosides, vol. 1804, pp. 19-55, 2018.

[8] S. Cavdarli, P. Delannoy, and S. Groux-Degroote, "O-acetylated gangliosides as targets for cancer immunotherapy," Cells, vol. 9, no. 3, p. 741, 2020. 
[9] G. Schwarzmann, "Labeled gangliosides: their synthesis and use in biological studies," FEBS Letters, vol. 592, no. 23, pp. 3992-4006, 2018.

[10] B. Liu, J. Ji, Q. Feng et al., "Monosialoganglioside protects against bupivacaine-induced neurotoxicity caused by endoplasmic reticulum stress in rats," Drug Design, Development and Therapy, vol. 13, pp. 707-718, 2019.

[11] V. Leonhard, R. V. Alasino, M. E. Pasqualini, D. C. Cremonezzi, N. H. García, and D. M. Beltramo, "Monosialoganglioside GM1 reduces toxicity of Ptx and increase anti-metastasic effect in a murine mammary cancer model," Scientific Reports, vol. 10, no. 1, Article ID 10191, 2020.

[12] A. Ranasinghe, J. Mehl, C. D’Arienzo et al., "Fucosyl monosialoganglioside: quantitative analysis of specific potential biomarkers of lung cancer in biological matrices using immunocapture extraction/tandem mass spectrometry," Rapid Communications in Mass Spectrometry, vol. 32, no. 17, pp. 1481-1490, 2018.

[13] E. Bazulin, "The maximum entropy method in ultrasonic nondestructive testing-increasing the resolution, image noise reduction and echo acquisition rate," Entropy, vol. 20, no. 8, p. $621,2018$.

[14] S. Shaoguo Cui and D. C. Liu, "Noise reduction for ultrasonic elastography using transmit-side frequency compounding: a preliminary study," IEEE Transactions on Ultrasonics, Ferroelectrics, and Frequency Control, vol. 58, no. 3, pp. 509-516, 2011.

[15] D. Shao, Y. Yuan, Y. Xiang, Z. Yu, P. Liu, and D. C. Liu, "Artifacts detection-based adaptive filtering to noise reduction of strain imaging," Ultrasonics, vol. 98, pp. 99-107, 2019.

[16] N. Damodaran, S. Ramamurthy, S. Velusamy, and G. Kanakaraj Manickam, "Speckle noise reduction in ultrasound biomedical B-scan images using discrete topological derivative," Ultrasound in Medicine and Biology, vol. 38, no. 2, pp. 276-286, 2012.

[17] Z. Lv, X. Li, and W. Li, "Virtual reality geographical interactive scene semantics research for immersive geography learning," Neurocomputing, vol. 254, no. 54, pp. 71-78, 2017.

[18] E. Chiricozzi, L. Mauri, G. Lunghi et al., "Parkinson's disease recovery by GM1 oligosaccharide treatment in the B4galnt1+/ - mouse model," Scientific Reports, vol. 9, no. 1, Article ID 19330, 2019.

[19] R. W. Ledeen and G. Wu, "Gangliosides, $\alpha$-synuclein, and Parkinson's disease,” Progress in Molecular Biology and Translational Science, vol. 156, pp. 435-454, 2018.

[20] X.-h. Ba, "Therapeutic effects of GM1 on Parkinson's disease in rats and its mechanism," International Journal of Neuroscience, vol. 126, no. 2, pp. 163-167, 2016.

[21] E. Di Biase, G. Lunghi, M. Maggioni et al., "GM1 oligosaccharide crosses the human blood-brain barrier in vitro by a paracellular route," International Journal of Molecular Sciences, vol. 21, no. 8, p. 2858, 2020.

[22] R. Paridar, M. Mozaffarzadeh, V. Periyasamy, M. Pramanik, M. Mehrmohammadi, and M. Orooji, "Sparsity-based beamforming to enhance two-dimensional linear-array photoacoustic tomography," Ultrasonics, vol. 96, pp. 55-63, 2019. 